\title{
Hammering home the sickle: an instructive case of endobronchial anaplastic large cell lymphoma
}

\author{
Mohammad Abu-Hishmeh', Laura Miranda ${ }^{2}$, Fouzia Shakil ${ }^{3}$, Tauseef Ahmed ${ }^{4}$, Oleg Epelbaum² \\ ${ }^{1}$ Division of Pulmonary, Critical Care, and Sleep Medicine, Baystate Medical Center, Springfield, MA; ${ }^{2}$ Division of \\ Pulmonary, Critical Care, and Sleep Medicine, Westchester Medical Center, Valhalla, NY; ${ }^{3}$ Department of Pathology, \\ Westchester Medical Center, Valhalla, NY; ${ }^{4}$ Division of Medical Oncology, Westchester Medical Center, Valhalla, NY, USA
}

\begin{abstract}
It is extremely unusual for primary pulmonary lymphoma, an uncommon occurrence in any form, to be of T-cell origin and to manifest as an endobronchial lesion. Each of these characteristics is rare individually, so cases that combine them are exceptional. We report a patient in whom primary pulmonary anaplastic large cell lymphoma, a T-cell neoplasm, presented with obstruction of the left upper lobe bronchus, resulting in left upper lobe atelectasis and creating the radiographic luftsichel sign. We briefly discuss anaplastic
\end{abstract}

Correspondence: Mohammad Abu Hishmeh, MD, Baystate Medical Center, 759 Chestnut St, Springfield, MA 01199, USA.

Tel. +001.413.794-3887 - Fax: +001.413.794-3987.

E-mail:memaad@hotmail.com

Key words: Primary pulmonary lymphoma; anaplastic large cell lymphoma; luftsichel sign.

Financial Support: None

Contributions: Each of the Authors participated in curation of literature, formulation of the manuscript from conception to design, analysis and finished product. OE played a supervisory role, as Senior Author, in the conception of the topic, editing, analysis, revision of intellectual content, and in literature review. The work has been reviewed and approved by all co- authors.

Conflict of interest: The Authors report no conflicts of interest.

Funding: The Authors do not report the receipt of any form of funding for the project.

Ethics approval: No ethical committee approval was required for this case report by the Department, because this article does not contain any studies with human participants or animals.

Received for publication: 7 September 2020.

Accepted for publication: 24 February 2021.

${ }^{\circ}$ Copyright: the Author(s), 2021

Licensee PAGEPress, Italy

Monaldi Archives for Chest Disease 2021; 91:1601

doi: 10.4081/monaldi.2021.1601

This article is distributed under the terms of the Creative Commons Attribution Noncommercial License (by-nc 4.0) which permits any noncommercial use, distribution, and reproduction in any medium, provided the original author(s) and source are credited. large cell lymphoma as a whole, place our case in the context of previously published literature on endobronchial anaplastic large cell lymphoma, and review the genesis of the luftsichel sign.

\section{Introduction}

In contrast to lung involvement by systemic lymphoma, primary pulmonary non-Hodgkin lymphoma (PPL), that is to say lymphoma arising directly from lung tissue, is an uncommon event and becomes even more remarkable when it is of T-cell origin. Anaplastic large cell lymphoma (ALCL) is a neoplasm of Tcell lineage the occurrence of which as PPL numbers only approximately 20 cases in total in the English-language literature [1]. The presentation of PPL of any type with proximal airway obstruction is rare, so endobronchial primary pulmonary ALCL as cause of lobar atelectasis is an exceptional diagnosis [2]. Herein we describe a case with this exact constellation and place it in the context of previously published reports. Additionally, we use this opportunity to also review the luftsichel sign, which is the radiographic finding that led to the discovery of our patient's unique endobronchial lesion.

\section{Case Report}

A 44-year-old man with no past medical history was referred to the pulmonary clinic of our institution for cough with occasional bloody sputum of one month's duration. Earlier evaluation elsewhere had included a chest radiograph (CXR), the interpretation of which indicated a normal film. There were no constitutional symptoms, and by the patient's account, the frequency of the hemoptysis episodes was waning. He was originally from Mexico and worked as a painter. He had no known history of tuberculosis and reported prior negativity of interferon gamma release assay for Mycobacterium tuberculosis. He had a minimal smoking history. Vital signs and physical examination were unremarkable. His presentation was attributed to antecedent viral infection and he was reassured about likely continued improvement; nevertheless, he was referred for a repeat CXR at our institution. Upon return one month later, he reported continued cough with episodic bloody sputum and new onset of dyspnea on exertion. CXR requested at the initial encounter was reviewed, and it showed left upper lobe (LUL) atelectasis accompanied by the luftsichel sign (Figure 1). Subsequent chest computed tomography without intravenous contrast confirmed LUL atelectasis and revealed an abrupt 

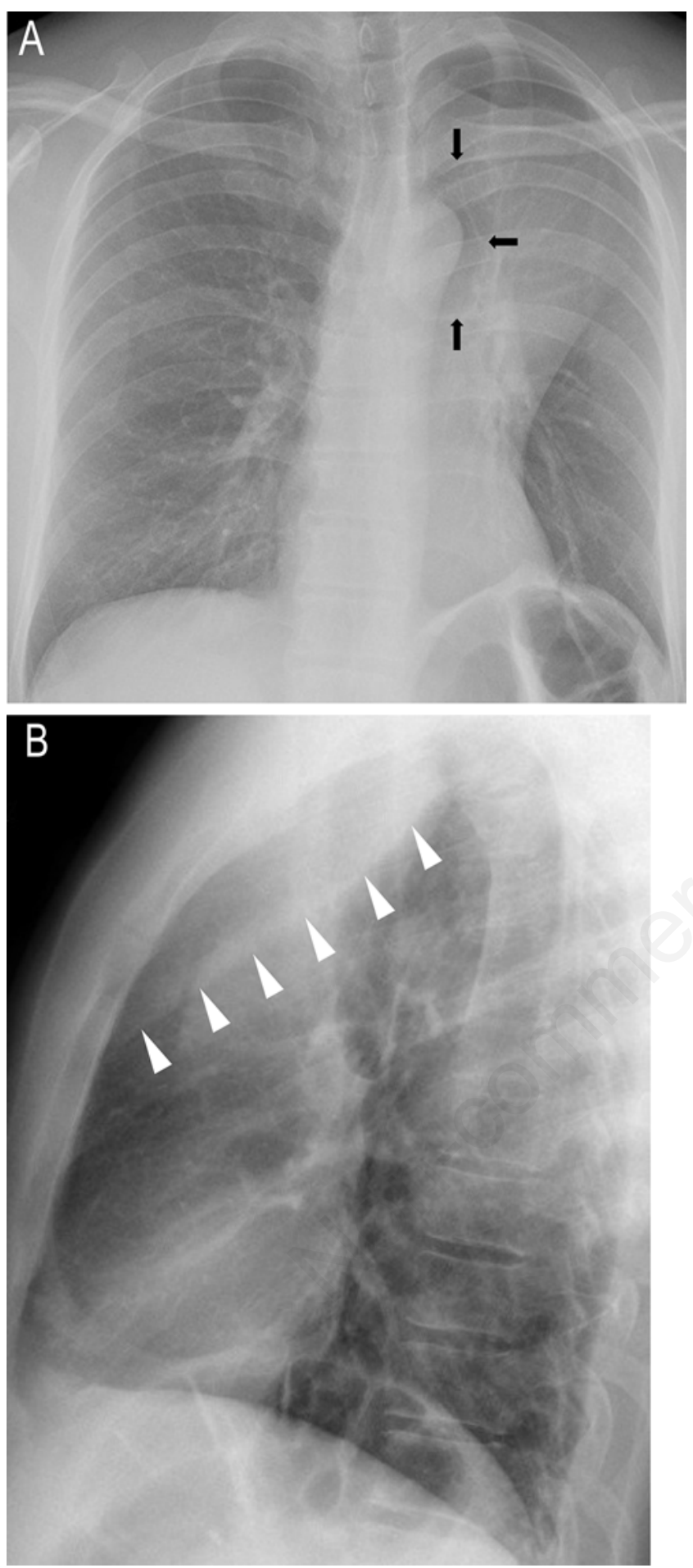

Figure 1. A) Frontal chest radiograph shows comp volume loss in the left hemithorax with a veil-like opacity in the upper lung field silhouetting the left heart border and corresponding to a collapsed left upper lobe. Luftsichel sign indicative of left upper lobe atelectasis is demarcated by arrows. Elevation and tenting of the left hemidiaphragm is present as an indirect sign of lobar collapse. B) Lateral chest radiograph confirms the attribution of the left upper lung field opacity seen on the frontal view to an atelectatic left upper lobe with resultant superoanterior displacement of the fissure (arrowheads). cut-off of the LUL bronchus due to endobronchial obstruction (Figure 2). These findings prompted flexible bronchoscopy, at which an amorphous mass-like structure was seen protruding from the orifice of the LUL bronchus (image not available). Microscopic sections of material obtained from this endoluminal lesion demonstrated infiltration by large, pleomorphic lymphoid cells with high nucleus:cytoplasm ratio, coarse chromatin, and prominent nucleoli, all pointing to malignancy of lymphoid origin (Figure 3). Some of the malignant cells exhibited horseshoeshaped nuclei, fitting the description of so-called "hallmark cells." Immunohistochemical staining was positive for CD4, CD5, CD30 and CD45 and negative for CD3, CD7, AE1/3, Napsin A, and TTF1 , identifying this as a malignancy of T-cell origin, specifically ALCL. Immunohistochemical staining for the anaplastic lymphoma kinase (ALK) was positive both in the nuclei and cyto-
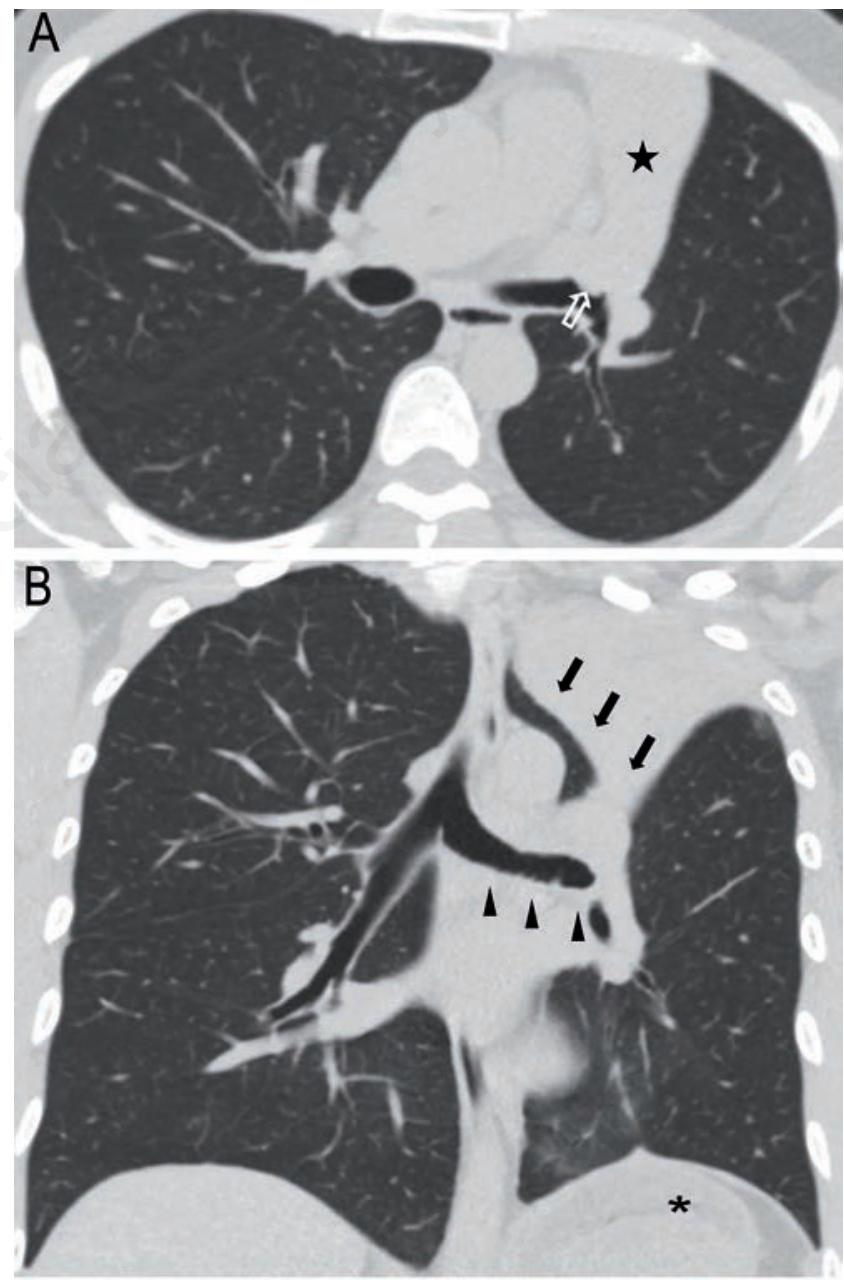

Figure 2. A) Axial image from computed tomography of the chest set to lung window demonstrates complete left upper lobe atelectasis (star) associated with an abrupt cut-off of the left upper lobe bronchus. An obstructing lesion can be seen protruding from the expected location of the origin of the left upper lobe bronchus (arrow). B) Coronal reconstruction image from computed tomography of the chest set to lung window highlights the crescentic lucency bordering the aortic knob that accounts for the radiographic luftsichel sign (arrows). Indirect signs of lobar collapse visible on this image include horizontal displacement of the left mainstem bronchus (arrowheads) as well as elevation and tenting of the left hemidiaphragm (asterisk). 
plasm, making this the ALK+ variant of ALCL. Endobronchial ultrasound-guided transbronchial needle aspiration of lymph node stations $4 \mathrm{~L}, 7$ and $10 \mathrm{~L}$ was negative for malignant cells. On 18Fluorodeoxyglucose positron emission tomography (18FDGPET), markedly increased 18FDG avidity (maximum standardized uptake value 26.13) was observed over a large area of the collapsed LUL, corresponding to the extent of lung tissue involvement by ALCL (Figure 4A). Non-enlarged, hypermetabolic mediastinal lymph nodes were also present, but there was no abnormal 18FDG uptake outside the thorax. Bone marrow sampling was negative for lymphoma, and no cytogenetic or genomic abnormalities were found. Following six cycles of cyclophosphamide, brentuximab, doxorubicin, and prednisone, the patient is considered to be in complete clinical remission based on post-treatment 18FDG- PET results (Figure 4B).

\section{Discussion}

PPL represents less than $1 \%$ of all non-Hodgkin lymphomas (NHL) and a mere $0.3 \%$ of all primary lung neoplasms [3]. Lymphoma is considered to be of primary lung origin if it (a) involves the parenchyma or central bronchi either unilaterally or bilaterally with or without secondary intrathoracic lymph node involvement and (b) there is no evidence of extrathoracic disease for three months following initial diagnosis [4]. This definition is meant to differentiate PPL from secondary lymphomatous lung involvement as may occur through either hematogenous spread or contiguous invasion by lymphoma originating in mediastinal or hilar lymph nodes [5]. As an extranodal lymphoma, PPL arises from bronchus-associated lymphoid tissue (BALT), which consists of a combination of B- and T-cells [3]. The more common but also less aggressive B-cell PPL is marginal zone lymphoma of BALT

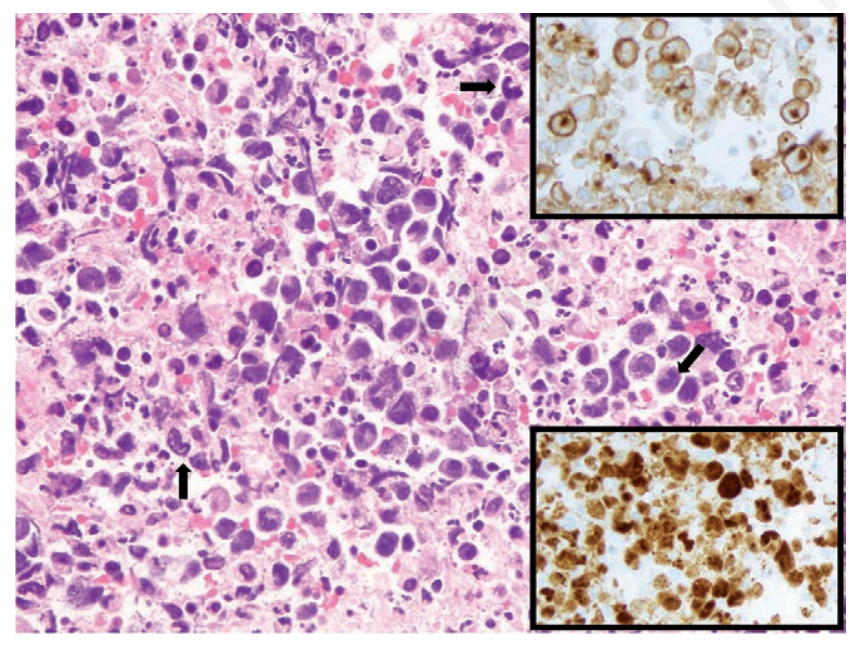

Figure 3. Tissue specimen obtained via bronchoscopic biopsy of the left upper lobe endobronchial lesion and viewed at high magnification (400X, hematoxylin $\&$ eosin) shows a population of pleomorphic lymphoid cells with high nucleus:cytoplasm ratio, coarse chromatin, occasional prominent nucleoli, and scattered cells with kidney- or horseshoe-shaped nuclei known as "hallmark" cells (arrows). This malignant histomorphological pattern favoring anaplastic large cell lymphoma (ALCL) is supported by positive immunohistochemical staining for CD30 in a membranous and Golgi pattern (inset top right). Strongly positive nuclear and cytoplasmic staining for anaplastic lymphoma kinase (ALK) identifies this as the ALK+ variant of ALCL (inset bottom right).
[6]. The less common but more aggressive type is diffuse large Bcell lymphoma. Our patient's ALCL, a form of T-cell PPL, is an exceedingly rare occurrence described only in individual case reports, which number very few if restricted to adult patients, and a single adult series [7]. Among the already scarce adult cases of ALCL presenting as PPL, our case occupies an even narrower niche limited to those with central bronchial obstruction by an endoluminal mass, as opposed to extrinsic compression, and with direct histological proof of ALCL as the etiology of obstruction provided (or at least documented) in the source publication. The application of these criteria placed our case in the company of only six others identified through a search of PubMed and Google Scholar [7-11].

The seven total cases are summarized and compared in Table 1. Certain distinguishing features of our case relative to the others deserve mention. It is one of four in which both CT images and explicit histological confirmation of endobronchial ALCL are available in the case description. It is the only one in which bronchoscopic biopsy established the diagnosis without the help of a surgical or bronchoscopic resection specimen. Although diagnosis in the case by Von Ende et al. [10] required pneumonectomy, its features are strikingly similar to ours, including patient characteristics, the availability of 18 FDG-PET to document localization of
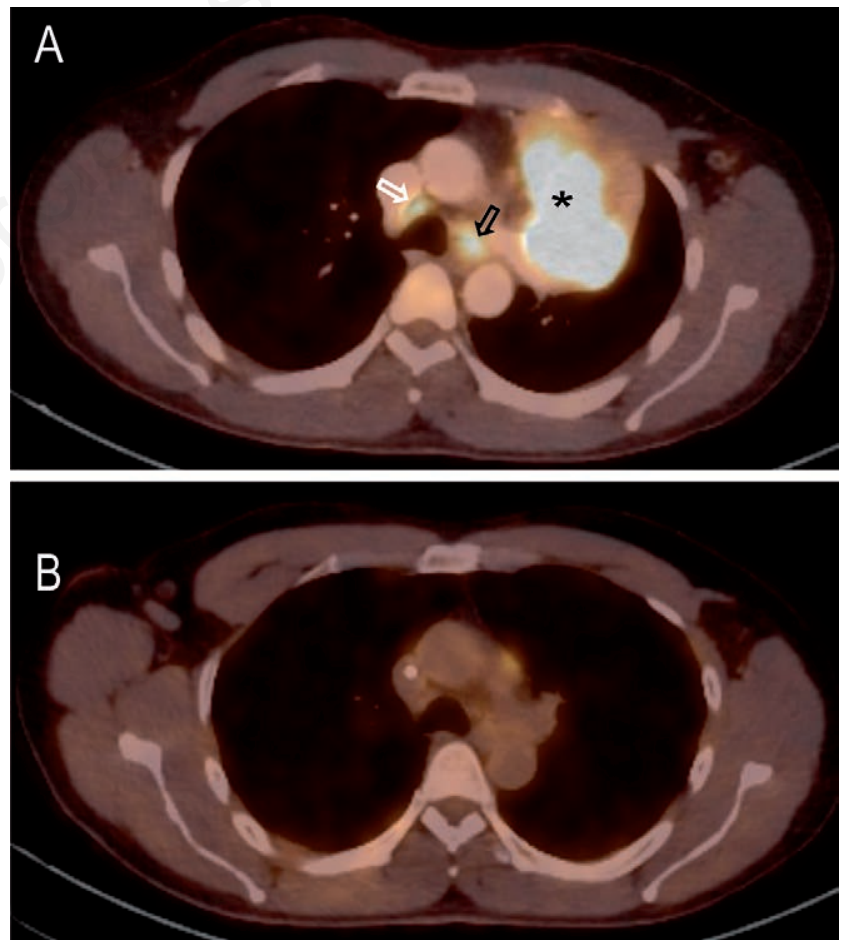

Figure 4. A) 18Fluorodeoxyglucose-positron emission tomography/computed tomography axial fusion image shows a large hypermetabolic focus (asterisk) corresponding to lymphomatous involvement of the collapsed left upper lobe (maximal standardized uptake value 26.13). Less intense but still hypermetabolic activity is seen in subcentimeter right paratracheal (white arrow) and left paratracheal (black arrow) mediastinal lymph nodes. B) 18Fluorodeoxyglucose-positron emission tomography/ computed tomography axial fusion image obtained upon completion of CHOP chemotherapy shows re-aeration of the left upper lobe and disappearance of the large hypermetabolic focus, previously seen within it, corresponding to the site of the patient's primary pulmonary lymphoma. 
Table 1. Summary of the seven published cases identified by the authors as representing intrinsic central endobronchial obstruction attributable to ALCL.

\begin{tabular}{|c|c|c|c|c|c|c|c|c|}
\hline Year[Ref] & Country & Age/sex & Location & $\mathrm{CT} * \mathrm{Y} / \mathrm{N}$ & Diagnostic procedure & Histology* Y/N & ALK & Outcome \\
\hline $1998[8]$ & SouthKorea & 28/M & LMB & Y & Bronchoscopic resection & Y & NR & Alive at 5 months \\
\hline $2000[7]$ & United States & $34 / \mathrm{M}$ & LLLbronchus & $\mathrm{N}$ & NR & Y & NR & Alive at 42 months \\
\hline $2000[7]$ & UnitedStates & $58 / \mathrm{F}$ & Trachea & $\mathrm{N}$ & NR & $\mathrm{Y}$ & $\mathrm{NR}$ & Dead at 6 months \\
\hline 2016[9] & India & 24/M & RMB & Y & Lobectomy & $\mathrm{Y}$ & + & NR \\
\hline $2018[10]$ & UnitedStates & $42 / \mathrm{M}$ & LULbronchus & $\mathrm{Y}$ & Pneumo-nectomy & $\mathrm{Y}$ & + & Alive at 6 months \\
\hline $2019[11]$ & Portugal & $85 / \mathrm{M}$ & LMB & $\mathrm{Y}$ & NR & $\mathrm{N}$ & + & Expired\# \\
\hline $2021^{\circ}$ & United States $^{\wedge}$ & $44 / \mathrm{M}$ & LULbronchus & $\mathrm{Y}$ & Bronchoscopic biopsy & $\mathrm{Y}$ & + & Alive at lyear \\
\hline
\end{tabular}

*Refers to whether the source publication contained CT images that documented intrinsic endobronchial obstruction and histological photomicrographs establishing ALCL as the cause of obstruction; \#timeline not specified; ^ patient originally from Mexico; ${ }^{\circ}$ current case; ALK, anaplastic lymphoma kinase; CT, computed tomography; LLL, left lower lobe; LMB, left mainstem bronchus; LUL, left upper lobe; NR, not reported; $\mathrm{RMB}$, right mainstem bronchus.

lymphoma to the thorax, and even the presence of the luftsichel sign as the initial radiographic clue to endobronchial obstruction. Translated from the German as "air sickle," luftsichel is a CXR sign of LUL atelectasis that refers to the crescentic lucency seen between the collapsed LUL and the aortic knob (Figure 1A) [12]. With complete LUL collapse, all aeration in the left hemithorax belongs to the left lower lobe (LLL). The luftsichel is created by the interposition of a sliver of air from the hyperinflated superior segment of the LLL between the aortic arch and the anteromedially displaced atelectatic LUL that is situated against the anterior chest wall abutting the left heart border (Figure 1B) [13]. To our knowledge, this is only the second case of the luftsichel sign attributed to lymphoma in the English-language literature. Of note, the other one is the aforementioned report by Von Ende et al, so the two cases share ALCL as the cause of LUL collapse. Overall, lymphoma is very rarely responsible for an endobronchial lesion, and among these exceptional cases extrinsic invasion by a mediastinal mass is more common than intrinsic obstruction by PPL [2].

In general, ALCL is a rare lymphoma of T-cell lineage that accounts for $<5 \%$ of adult NHL and typically afflicts young people like our patient [14]. Extranodal disease is common, but, as mentioned, the lung is seldom involved. It is characterized microscopically by large neoplastic lymphoid cells, some with distinct horseshoe- or kidney-shaped nuclei that have been termed "hallmark cells (Figure 3)." The immunohistochemical signature of ALCL is strongly positive staining for CD30 often accompanied by loss of T-cell markers such as CD3 by these poorly- differentiated cells, which is also true of our case [15]. The majority of ALCL exhibits autonomous expression of the oncogenic ALK protein (so-called $\mathrm{ALK}+\mathrm{ALCL}$ ), a tyrosine kinase whose synthesis is normally regulated, that results from a chromosomal translocation whereby the gene coding for ALK fuses with that of a ubiquitous partner, leading to uncontrolled ALK activity [16]. The most common rearrangement pairs the ALK gene with that of nucleophosmin, a constitutively expressed protein that shuttles between nucleus and cytoplasm, thus accounting for the mixed nuclear and cytoplasmic pattern of ALK staining in most patients, including ours [15]. ALK positivity is associated with a superior prognosis in ALCL compared to the less common ALK- variant: 5-year survival of 70\% vs. $49 \%$, though both figures are favorable compared to most other Tcell lymphomas [17]. The cornerstone of ALCL treatment is anthracycline-based chemotherapy, classically CHOP (cyclophosphamide, doxorubicin, vincristine, and prednisone). Substitution of the anti-CD30 agent brentuximab for vincristine, which is the regimen our patient received, has recently been associated with improved survival compared to CHOP [18]. Rapid and durable response of ALK+ ALCL to the ALK inhibitor crizotinib has been described in a small series [19] and trial [20].

\section{Conclusions}

PPL of any type as a cause of endobronchial obstruction is rare, even more so when the PPL itself is uncommon as was true of our patient's ALCL. This case not only highlights a rare form of PPL but also provides an opportunity to revisit an important sign in chest radiography, namely the luftsichel sign indicative of LUL collapse. The presence of this sign should always prompt a search for an endobronchial lesion, which in a young patient at low risk for primary lung cancer could lead to a surprising discovery, as it did in our case.

\section{Referneces}

1. Pan Z, Xu ML. T-cell and NK-cell lymphomas in the lung. Semin Diagn Pathol 2020;37:273- 82.

2. Solomonov A, Zuckerman T, Goralnik L, et al. Non-Hodgkin's lymphoma presenting as an endobronchial tumor: report of eight cases and literature review. Am J Hematol 2008;83:416-9.

3. Kligerman SJ, Franks TJ, Galvin JR. Primary extranodal lymphoma of the thorax. Radiol Clin North Am 2016;54:673-87.

4. L'Hoste RJ Jr, Filippa DA, Lieberman PH, Bretsky S. Primary pulmonary lymphomas. A clinicopathologic analysis of 36 cases. Cancer 1984;54:1397-406.

5. Cadranel J, Wislez M, Antoine M. Primary pulmonary lymphoma. Eur Respir J 2002;20:750- 62.

6. Sammassimo S, Pruneri G, Andreola G, et al. A retrospective international study on primary extranodal marginal zone lymphoma of the lung (BALT lymphoma) on behalf of International Extranodal Lymphoma Study Group (IELSG). Hematol Oncol 2016;34:177-83.

7. Rush WL, Andriko JA, Taubenberger JK, et al. Primary anaplastic large cell lymphoma of the lung: a clinicopathologic study of five patients. Mod Pathol 2000;13:1285-92.

8. Kim DH, Ko YH, Lee MH, Ree HJ. Anaplastic large cell lymphoma presenting as an endobronchial polypoid mass. Respiration 1998;65:156-8. 
9. Chand T, Bansal A, Dua H, Sharma K. Endotracheobronchial lymphoma: Two unusual case reports and review of article. Lung India 2016;33:653-6.

10. Von Ende E, Kauffman T, Munoz PA, Martinez-Jiménez S. Primary pulmonary anaplastic large cell lymphoma: A rare malignancy and rare cause of the luftsichel sign. Case Rep Radiol 2018;2018:8574642.

11. Carvalho J, Marques DP, Oliveira I, Claudino C. A rare case of primary pulmonary anaplastic large cell lymphoma. Eur J Case Rep Intern Med 2019;6:001249.

12. Blankenbaker DG. The luftsichel sign. Radiology 1998;208:319-20.

13. Day K, Oliva I. Signs in cardiopulmonary imaging: luftsichel sign. J Thorac Imaging 2015;30:W1.

14. Eyre TA, Khan D, Hall GW, Collins GP. Anaplastic lymphoma kinase-positive anaplastic large cell lymphoma: current and future perspectives in adult and paediatric disease. Eur $\mathrm{J}$ Haematol 2014;93:455-68.

15. Leventaki V, Bhattacharyya S, Lim MS. Pathology and genet- ics of anaplastic large cell lymphoma. Semin Diagn Pathol 2020;37:57-71.

16. Chiarle R, Voena C, Ambrogio C, et al. The anaplastic lymphoma kinase in the pathogenesis of cancer. Nat Rev Cancer 2008;8:11-23.

17. Vose J, Armitage J, Weisenburger D, International T-Cell Lymphoma Project. International peripheral T-cell and natural killer/T-cell lymphoma study: pathology findings and clinical outcomes. J Clin Oncol 2008;26:4124-30.

18. Horwitz S, O'Connor OA, Pro B, et al. Brentuximab vedotin with chemotherapy for CD30- positive peripheral T-cell lymphoma (ECHELON-2): a global, double-blind, randomised, phase 3 trial. Lancet 2019;393:229-40. Erratum in: Lancet 2019;393:228.

19. Gambacorti-Passerini C, Messa C, Pogliani EM. Crizotinib in anaplastic large-cell lymphoma. N Engl J Med 2011;364:775-6.

20. Gambacorti Passerini C, Farina F, Stasia A, et al. Crizotinib in advanced, chemoresistant anaplastic lymphoma kinase-positive lymphoma patients. J Natl Cancer Inst 2014;106:djt378. 